Supporting Information for

\title{
Multiplexed Detection of Epigenetic Markers Using Quantum Dot (QD)-Encoded Hydrogel Microparticles
}

\author{
Sang Yun Yeom ${ }^{1,2 \#, ~ C h o o n g ~ H y u n ~ S o n ~}{ }^{1}$, Byung Sun Kim ${ }^{3,4,5}$, Sung Hyun Tag ${ }^{3,4}$, \\ Eunjoo Nam ${ }^{4}$, Hyogeun Shin ${ }^{1,6}$, So Hyun Kim ${ }^{1}$ Haemin Gang ${ }^{1}$, Hyunjoo J. Lee ${ }^{1,6}$, \\ Jungkyu Choi ${ }^{2,8 *}$, Heh-In Im $^{3,4,5 *}$, Il-Joo Cho ${ }^{1,6 *}$, and Nakwon Choi ${ }^{1,6 *}$
}

${ }^{1}$ Center for BioMicrosystems, Brain Science Institute, Korea Institute of Science and Technology (KIST), Seoul 02792, Korea

${ }^{2}$ Department of Chemical and Biological Engineering, Korea University, Seoul 02841, Korea

${ }^{3}$ Convergence Research Center for Diagnosis, Treatment and Care System of Dementia, Korea Institute of Science and Technology (KIST), Seoul 02792, Korea

${ }^{4}$ Center for Neuroscience, Brain Science Institute, Korea Institute of Science and Technology (KIST), Seoul 02792, Korea

${ }^{5}$ Department of Neuroscience, Korea University of Science and Technology (UST), Daejeon 34113, Korea

${ }^{6}$ Department of Biomedical Engineering, Korea University of Science and Technology (UST), Daejeon 34113, Korea

${ }^{7}$ School of Electrical Engineering, Korea Advanced Institute of Science and Technology (KAIST), Daejeon 34141, Korea

${ }^{8}$ Green School, Korea University, Seoul 02841, Korea

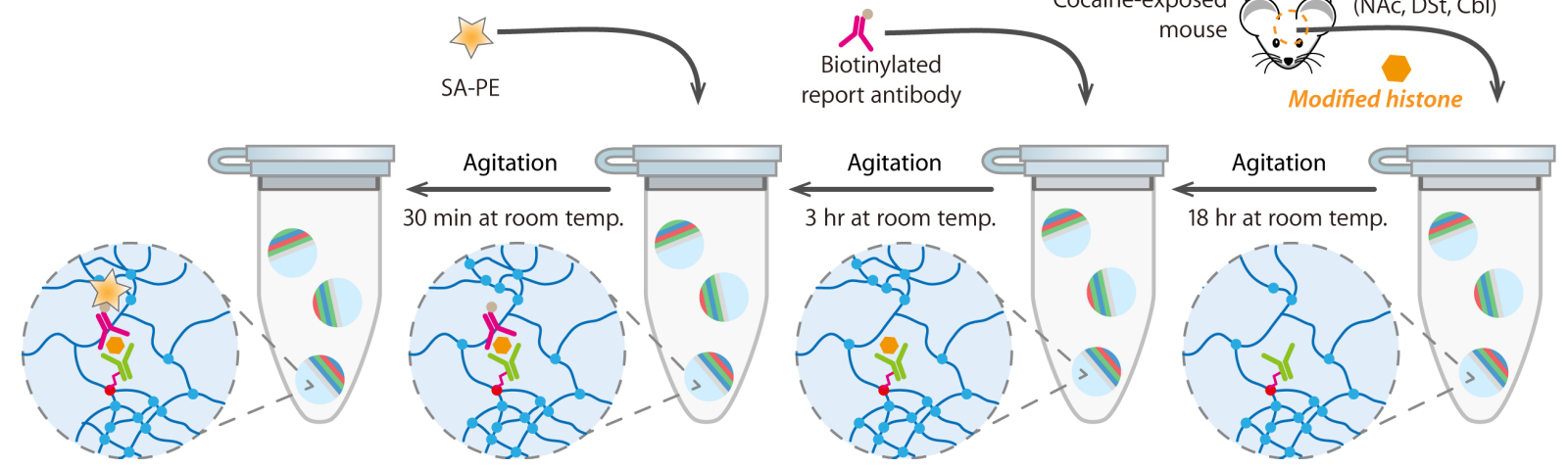

Figure S1. Detailed assay scheme for multiplexed detection of modified histones from mouse brain lysates. 1) incubation with a brain lysate per mouse containing 3 modified histones (NAc, DSt, or $\mathrm{Cbl}), 2$ ) incubation with a biotinylated report antibody, and 3) incubation with a reporter fluorophore of SA-PE. 


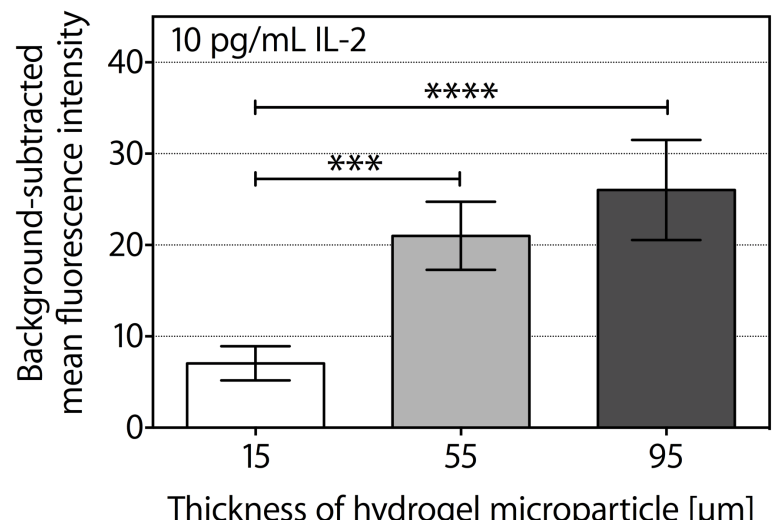

Figure S2. Optimization of thickness of hydrogel microparticles. Bar graphs presenting backgroundsubtracted mean fluorescence intensity of IL-2 $(10 \mathrm{pg} / \mathrm{mL})$ from hydrogel microparticles with thickness of 15 (white), 55 (gray), and 95 (dark gray) $\mu \mathrm{m} .5$ hydrogel microparticles were analyzed for each data point. Error bars represent standard deviation. ${ }^{* * *}$ and ${ }^{* * *}$ denote significant differences of $p<0.0001$ and $p<0.001$, respectively.

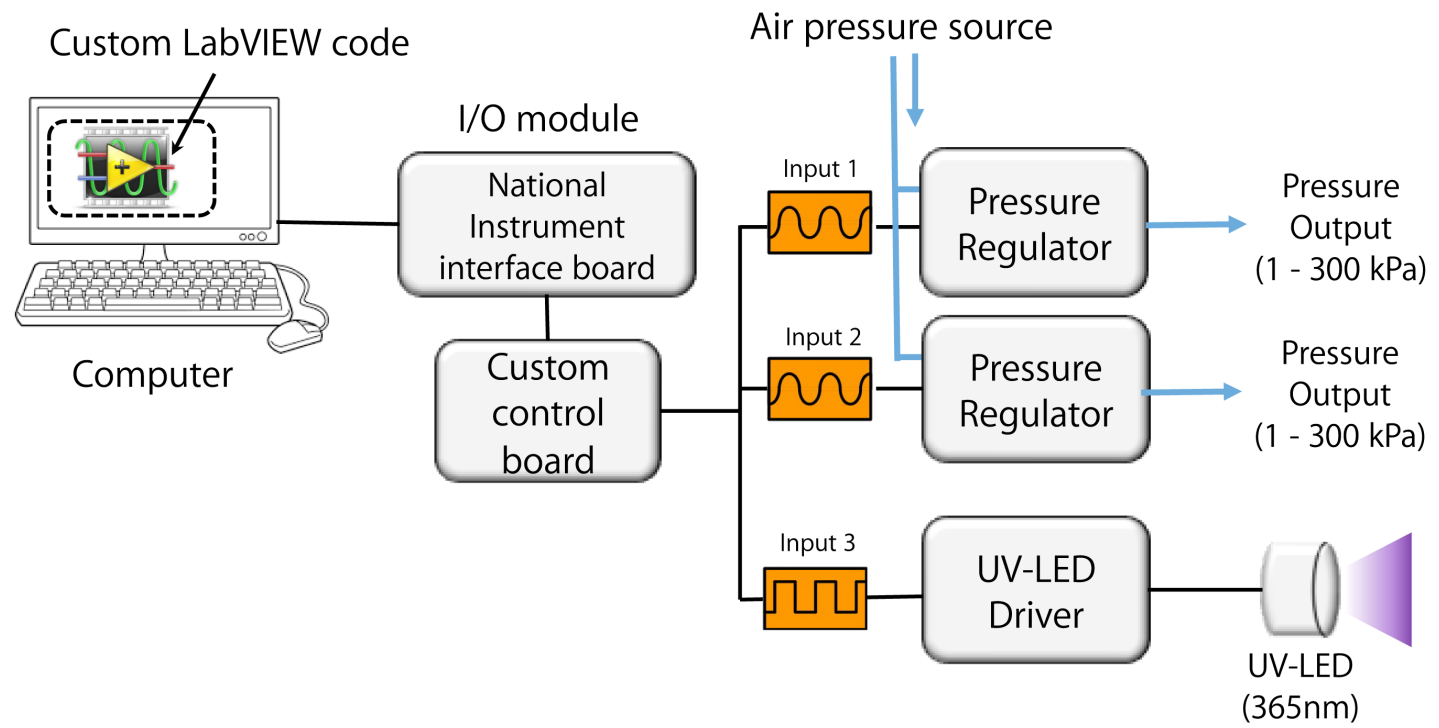

Figure S3. Block diagram of circuit design to control both pressure regulators and UV LED. Computer sends commands to a custom control board through the interface board to generate signals. Two analog signals and one digital signal are generated to control pressure regulators and UV-LED driver, respectively.

Table S1. Width of QD stripes by controlling pressure to probe and code regions

\begin{tabular}{cccccc}
\hline$P_{\text {probe }}$ & $P_{\text {code }}$ & $P_{\text {probe }} / P_{\text {code }}$ & $\begin{array}{c}w_{\text {QD-stripe,mean }} \\
{[\mu \mathrm{m}]}\end{array}$ & $\begin{array}{c}w_{\text {QD-stripe,SD }} \\
{[\mu \mathrm{m}]}\end{array}$ & $\begin{array}{c}\text { Coefficient of variation } \\
(\mathrm{CV})[\%]\end{array}$ \\
\hline 30 & 30 & 1 & 20.6 & 1.37 & 6.64 \\
45 & 15 & 3 & 15.2 & 1.08 & 7.09 \\
100 & 30 & 3.3 & 14.5 & 1.12 & 7.75 \\
50 & 10 & 5 & 11.3 & 1.05 & 9.31 \\
50.25 & 7.5 & 7 & 8.31 & 0.97 & 11.7 \\
104 & 6 & 9 & 6.25 & 0.81 & 12.9 \\
\hline
\end{tabular}




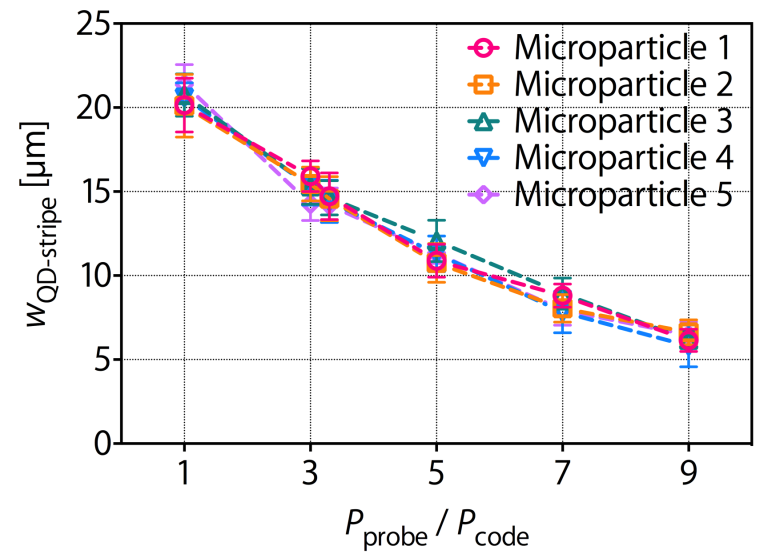

Figure S4. Inter- and intra-particle variations of width of QD-stripes by controlling ratio of pressure to probe and code regions. Red circle, organe square, green triangle, blue inverse triangle, and purple lozenge indicate mean of 5 QD-stripes per representative microparticle 1,2, 3, 4, and 5, respectively. Error bars represent standard deviation.
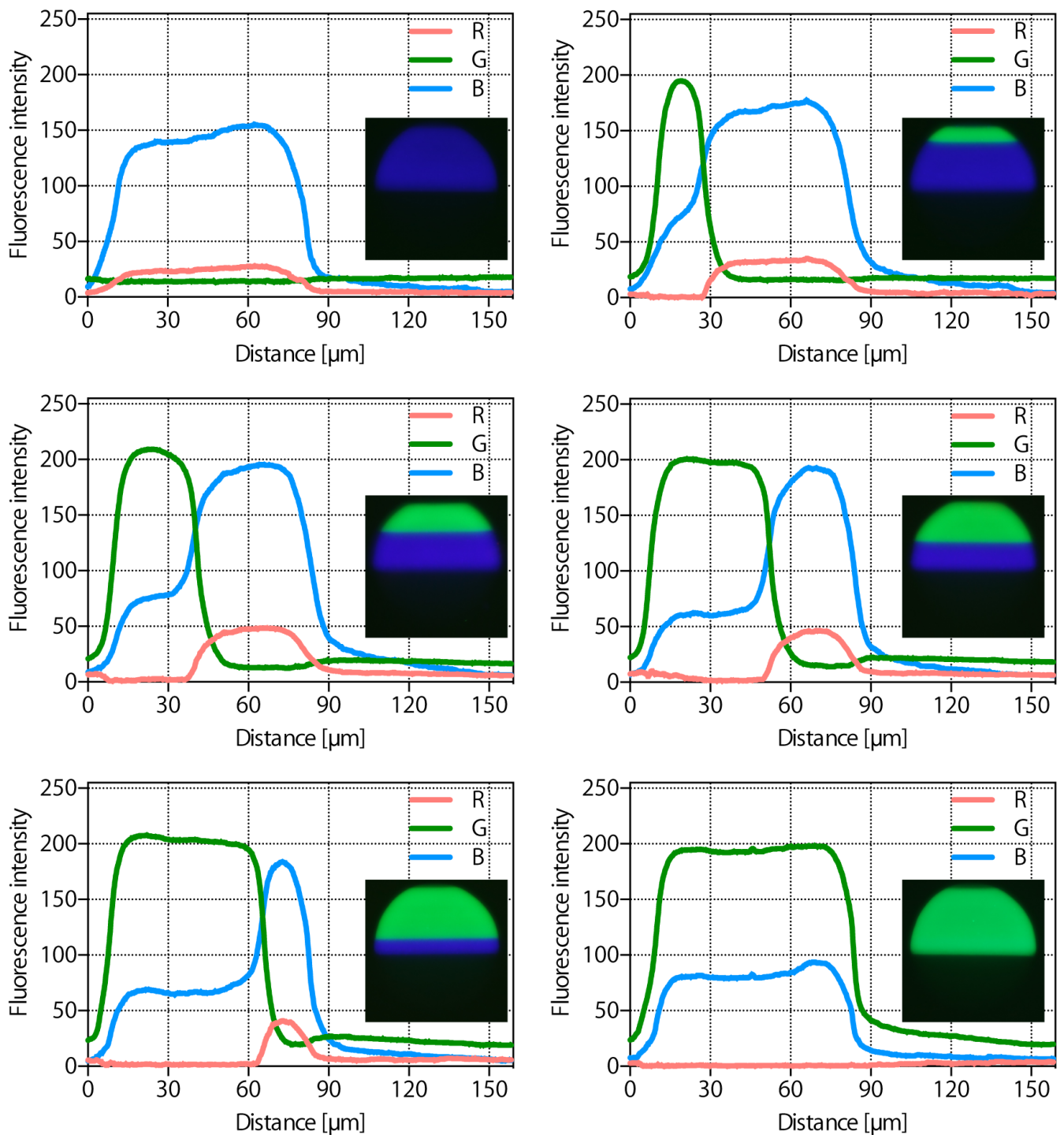

Figure S5. Spatial intensity profiles of red, green, and blue channels from representative microparticles (inset fluorescence micrographs) encoded with identical green and blue stripes consecutively located in the code region. 

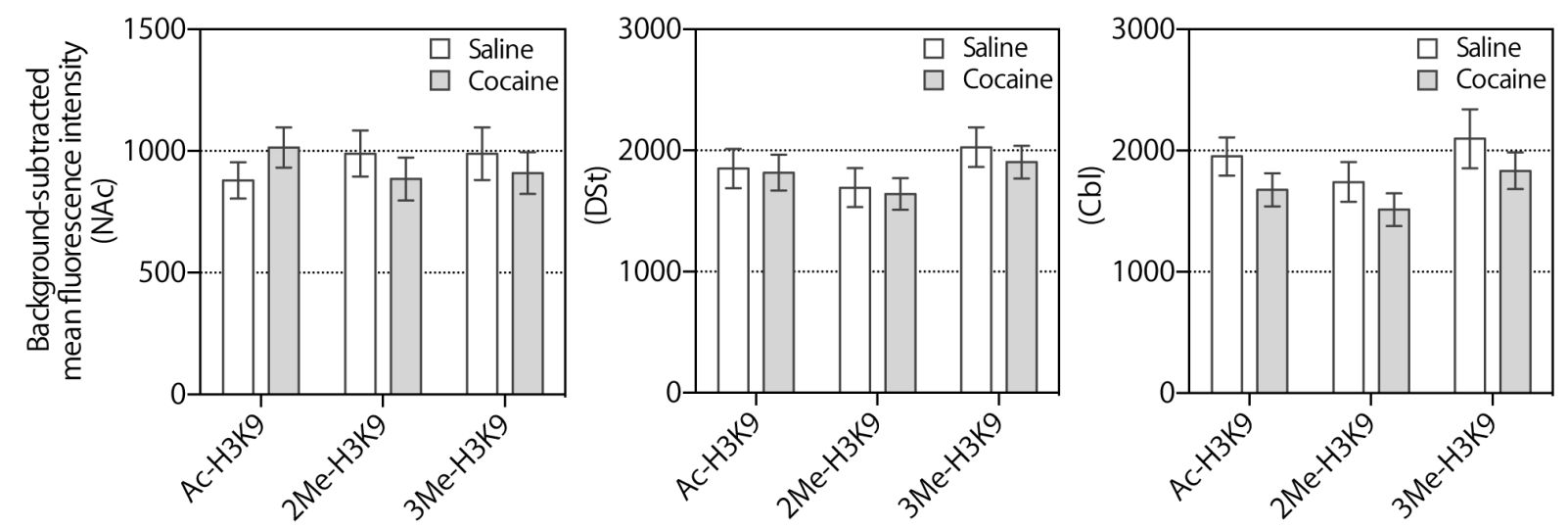

Figure S6. Representative example displaying detection of histone H3K9 modifications from mixture of multiple brain lysates. Bar graphs presenting background-subtracted mean fluorescence intensity of Ac-H3K9, 2Me-H3K9, and 3Me-H3K9 simultaneously detected from NAc (left), DSt (middle), and $\mathrm{Cbl}$ (right) of mice exposed to saline or cocaine for 7 days. White and gray bars are background-subtracted mean fluorescence intensity from saline- and cocaine-exposed groups, respectively. Error bars represent standard error of mean.
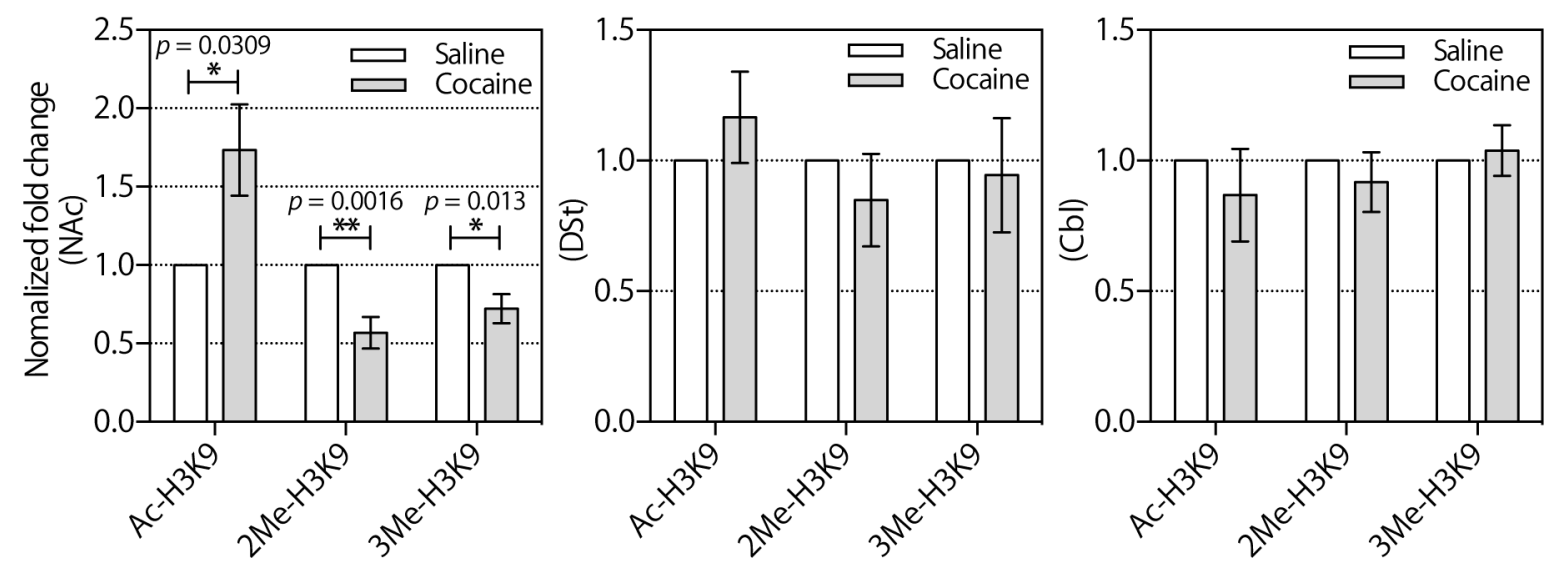

Figure S7. Western blot analysis from $50 \mu \mathrm{g}$ of total proteins in the brain lysates of cocaine-exposed mice. Normalized fold changes in H3K9 modifications from NAc (left), DSt (middle), and Cbl (right). Cocaine-exposed mice display significant elevation in Ac-H3K9 and reduction in Me2- \& Me3-H3K9 in response to cocaine. In both DSt and Cbl, no significant changes were observed. $\mathrm{n} \geq$ 4 mice per group. 

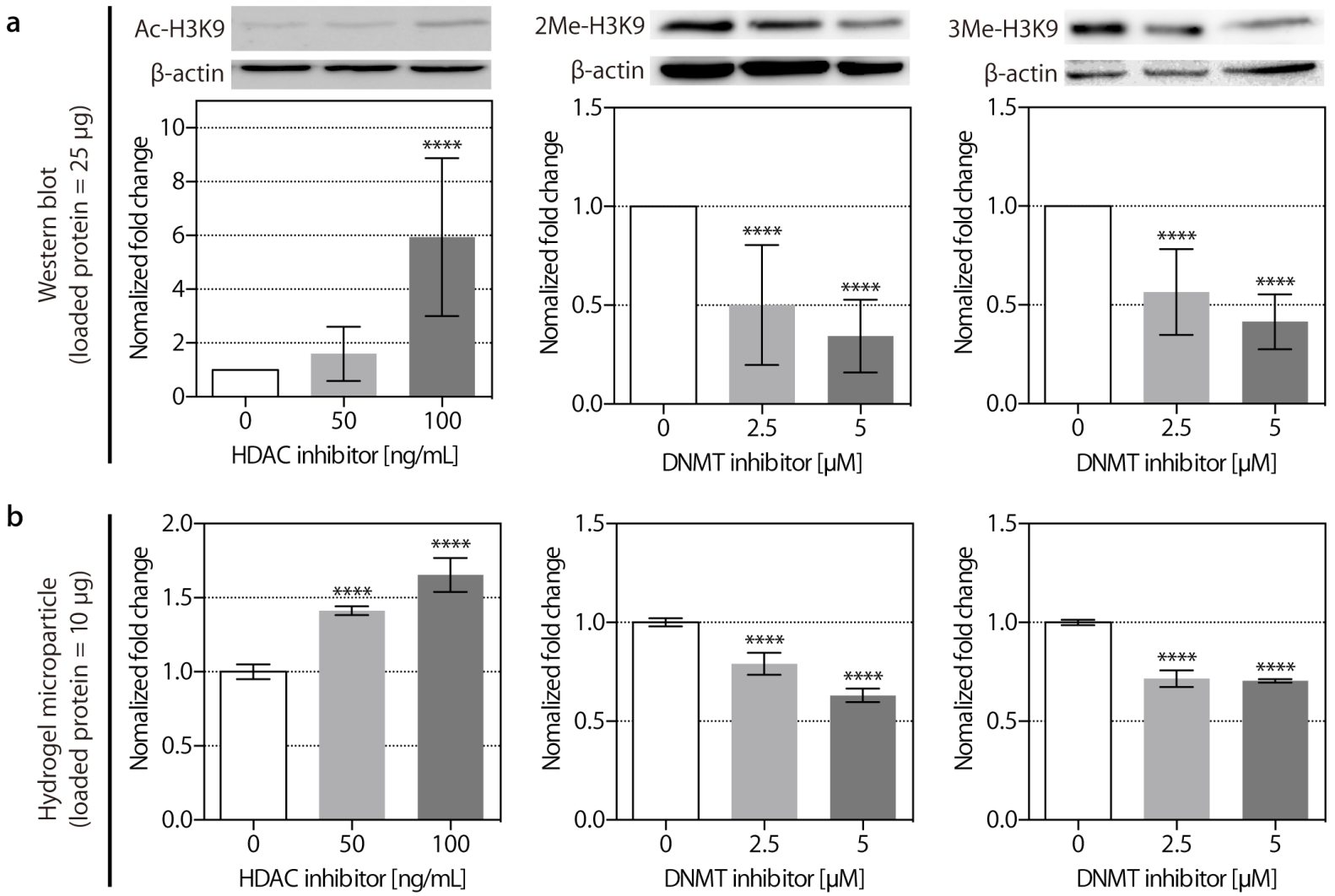

Figure S8. Quantification of histone H3K9 modifications from lysates of HEK cells treated with HDAC inhibitor or DNMT inhibitor. a. Western blot analysis with $25 \mu \mathrm{g}$ of total proteins. HDAC inhibitor dose-dependently increased Ac-H3K9 status, while DNMT inhibitor also dosedependently reduced $2 \mathrm{Me}-\mathrm{H} 3 \mathrm{~K} 9$ and $3 \mathrm{Me}-\mathrm{H} 3 \mathrm{~K} 9 .{ }^{* * * *}$ denotes $p<0.0001 . \mathrm{n}=3$ per group. $\mathrm{b}$. Plots of normalized fold changes for hydrogel microparticle-based assay with the identical lysates $(10 \mu \mathrm{g}$ of total proteins). ${ }^{* * *}$ denotes $p<0.0001$.

Video S1. Video clip showing microfluidic capture of hydrogel microparticles. 


\section{MATLAB code to rotate and crop acquired images}

clc; \%Clear Command Window

clear all; \%Remove items from workspace, freeing up system memory

close all;

\%Open standard dialog box for selecting directory

dname_fl = uigetdir('any desired location on a computer to find images' );

\%List all tif files

cd(dname fl);

img_path_fl=strcat(dname_fl,'/*.TIF');

file_fl $=$ dir(img_path_fl);

file_num_fl $=$ length $\left(\overline{f i l e} \_f l\right)$;

file_name_fl $=\{$ file_fl.name $\}$;

file_date_fl $=\{$ file_fl.date $\}$;

file num fl

clims_fl=[100 700];

rot_degree $=[]$;

upperleftcorner $=[]$;

img_w $=620$;

img_h=620;

probe_w $=550$;

probe_h$=250$;

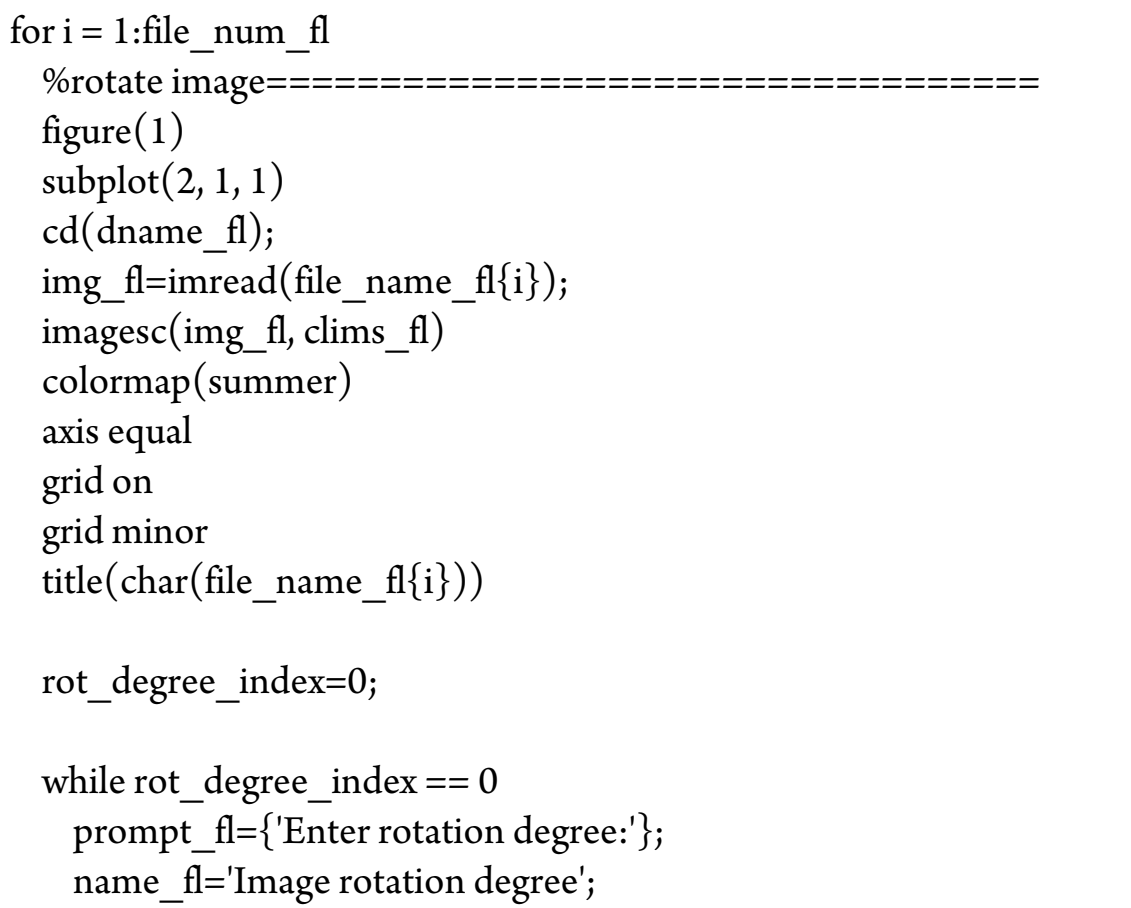




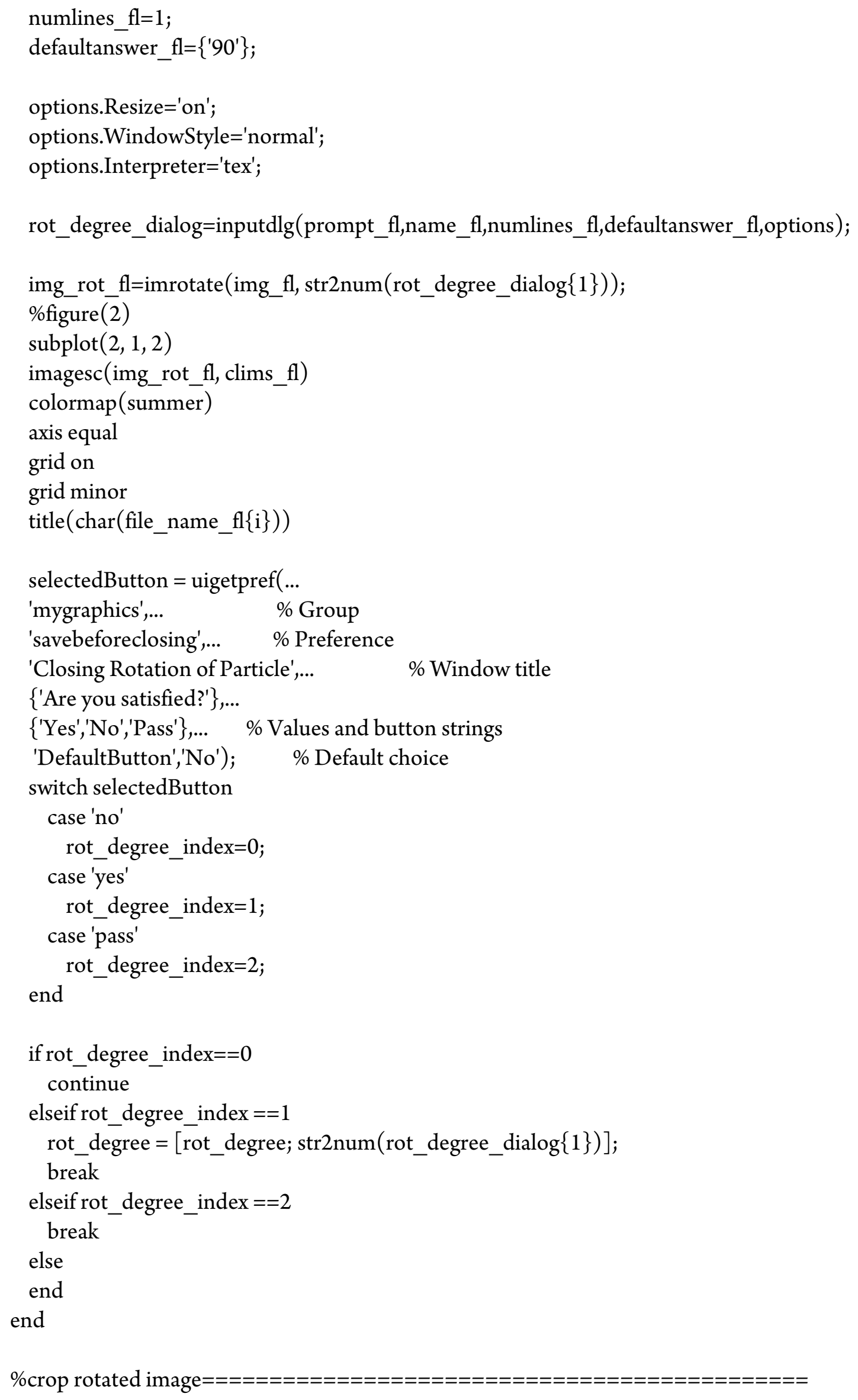




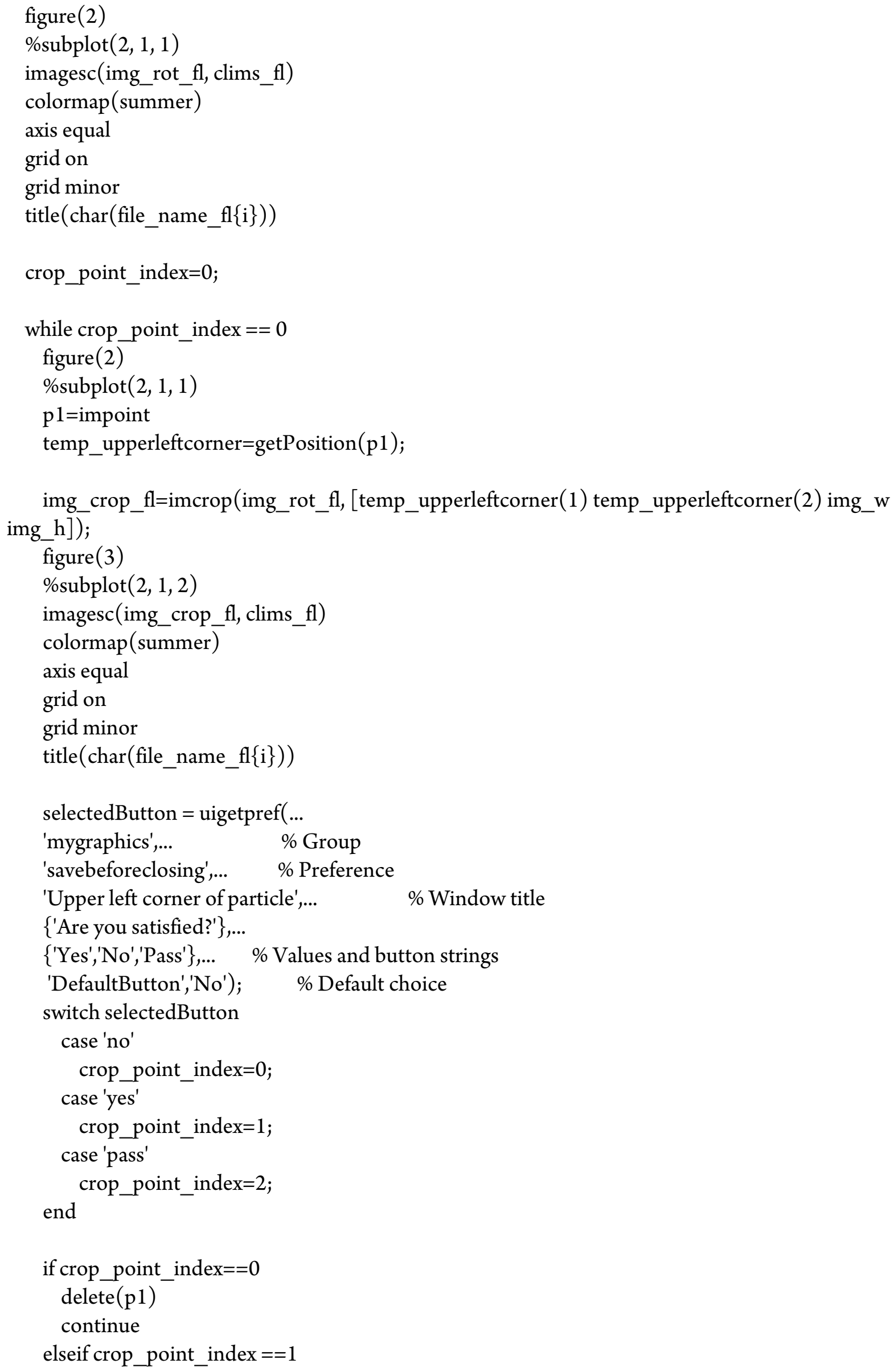




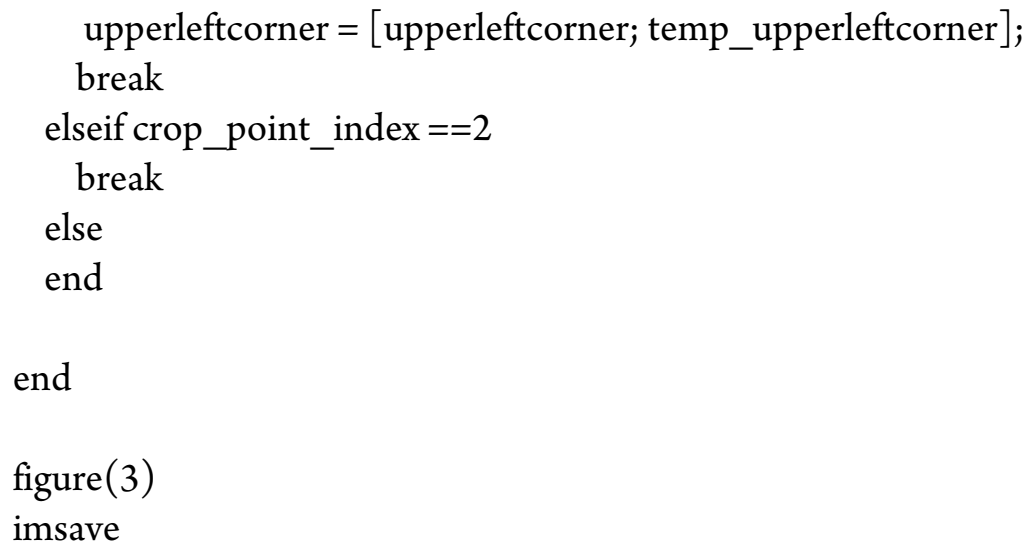




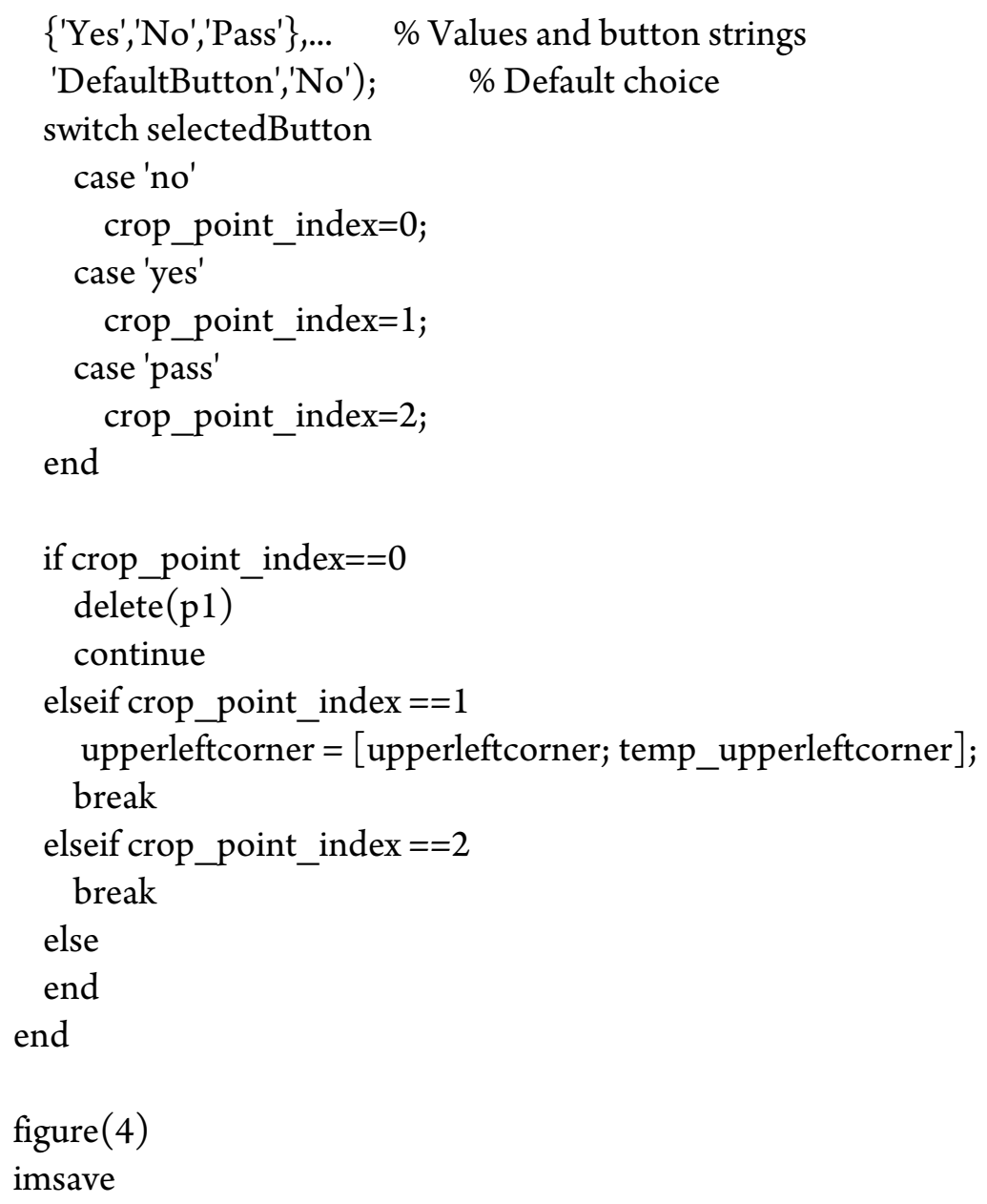




\section{MATLAB code to analyze fluorescence intensity from (rotated and cropped) images}

clc; \%Clear Command Window

clear all; \%Remove items from workspace, freeing up system memory

close all;

\%Open standard dialog box for selecting directory

dname = uigetdir('any desired location on a computer to find images');

\%List all tif files

cd(dname);

img_path=strcat(dname, $/^{*}$.tif $)$;

file $=\operatorname{dir}($ img_path $)$;

file_num = length(file);

file_name $=\{$ file.name $\}$;

file_date $=\{$ file.date $\}$

file_num

$\%$ Determine row \& column numbers for subplot

prompt $1=\{$ 'Enter row number:', 'Enter column number:' $\}$;

name1='Input for Limits of Fluorescence Intensity';

numlines $1=1$;

defaultanswer $1=\left\{5^{\prime},{ }^{\prime} 1 '\right\}$;

options.Resize='on';

options.WindowStyle='normal';

options.Interpreter='tex';

rowcolumn_dialog=inputdlg(prompt1,name1,numlines1,defaultanswer1,options);

subplotsize $=[\operatorname{str} 2$ num $($ rowcolumn_dialog $\{1,1\}) \operatorname{str} 2$ num $($ rowcolumn_dialog $\{2,1\})]$;

\%Determine limits of fluorescence intensity

prompt $2=\{$ 'Enter the lower limit of fluorescence intensity:', 'Enter the upper limit of fluorescence intensity:'\};

name2='Input for Limits of Fluorescence Intensity';

numlines $2=1$;

defaultanswer2 $=\{200$ ','13000' $\}$;

options.Resize $=$ 'on';

options.WindowStyle='normal';

options.Interpreter='tex';

clims_dialog=inputdlg(prompt2,name2,numlines2,defaultanswer2,options);

clims $=\left[\operatorname{str} 2\right.$ num $\left(\operatorname{clims} \_\operatorname{dialog}\{1,1\}\right) \operatorname{str} 2$ num $\left.\left(\operatorname{clims} \_\operatorname{dialog}\{2,1\}\right)\right]$ 
probe_sig=[];

\%Generate color-mapped images with the fixed limits for all images

figure()

for $\mathrm{i}=1$ :file_num

img=imread(file_name $\{\mathrm{i}\})$;

colormap (jet);

subplot(subplotsize(1), subplotsize(2),i);

imagesc(img, clims)

axis equal

axis off

\%colorbar

probe_sig=[probe_sig; mean $(\operatorname{mean}(\mathrm{img}))]$;

end

mean_probe_sig=mean(probe_sig)

stdev_probe_sig=std(probe_sig)

CV_probe_sig=stdev_probe_sig/mean_probe_sig* 100 